\title{
The Reservosome of Trypanosoma cruzi Epimastigotes: an Organelle of the Endocytic Pathway with a Role on Metacyclogenesis
}

\author{
Maurilio José Soares \\ Laboratório de Biologia Celular de Microrganismos, Departamento de Ultra-estrutura e Biologia Celular, \\ Instituto Oswaldo Cruz, Av. Brasil 4365, 21045-900 Rio de Janeiro, RJ, Brasil
}

Key words: reservosome - Trypanosoma cruzi - endocytosis - metacyclogenesis

Reservosomes are large $(0.4-0.6 \mu \mathrm{m}$ in diameter) membrane-bound organelles found at the posterior end of Trypanosoma cruzi epimastigote forms. They present a protein-rich, electron dense matrix, where several tiny round electron lucent inclusions are immersed. Due to their inner structure, these organelles were formerly designated as multivesicular bodies (De Souza 1984). However, ultrastructural demonstration that the inner inclusions were not membrane bound, together with cytochemical evidentiation that these vesicles were indeed lipid droplets, revealed the misinterpretation of the former nomenclature, and the name reservosome was then proposed (Soares \& De Souza 1988). Reservosomes are morphologically and biochemically distinct from the recently described acidocalcisomes (Scott et al. 1997). As reservosomes are a site for protein accumulation, two major questions arise: how proteins arrive there, and what reservosomes are for?

\section{THE ENDOCYTIC PATHWAY}

Endocytosis of nutrients in trypanosomatid protozoa is restricted to the flagellar pocket membrane (Webster \& Russel 1993, Radek \& Hausmann 1994, Overath et al. 1997). However, epimastigotes of $T$. cruzi present an additional site for the uptake of macromolecules: the cytostome, a deep invagination of the cell plasma membrane close to the flagellar pocket region. It appears that the cytostome is physically linked to the flagellum (Okuda et al. 1977) and represents the main site for both receptor-mediated-endocytosis and fluidphase-pinocytosis in epimastigotes (Soares \& De Souza 1991, Porto-Carreiro et al. 1998). Endocytic vesicles bud off from the cytostome and the flagellar pocket membranes and then deliver their cargo

Fax: +55-21-260.4434. E-mail: maurilio@ioc.fiocruz.br Received 9 June 1999

Accepted 9 August 1999 to the reservosomes (Soares \& De Souza 1991, Soares et al. 1992). Reservosomes contain tyrosinephosphorylated proteins, suggesting that protein kinases play a role in the internalization process (Vieira et al. 1996). Incubation of epimastigotes with ATP $(50 \mathrm{mM}$, for $24 \mathrm{hr})$ prior to the addition of horseradish peroxidase (as a marker of the endocytic pathway) affected the formation of normal reservosomes (Bogitsh et al. 1997), possibly acting on the (still unknown) translocation system governing the traffic of the small endocytic vesicles that cargo proteins from the cell surface to the storage organelles.

Reservosomes are acidic organelles, containing cruzipain (a cysteine proteinase) and ingested proteins. On this basis, it has been proposed that these structures are pre-lysosomal compartments (Soares et al. 1992). Imunocytochemical quantification using DAMP as a probe showed that reservosomes have a luminal $\mathrm{pH}$ of about 6.0 (Soares et al. 1992). However, it is still a matter of speculation how these organelles are acidified, as they were not labeled with antibodies against a vacuolar-type $\mathrm{H}^{+}$-ATPase, which however recognized other intracellular vacuoles, possibly the acidocalcisomes (Benchimol et al. 1998). A 52$\mathrm{kDa}$ protein sharing sequence homology with glutathione S-transferase (Tc52) has been also localized in reservosomes (Ouassi et al. 1995). It has been postulated that $\mathrm{Tc} 52$ is released from the parasite to the external milieu, in order to scavenge glutathione (GSH). The Tc52-GSH complex could be then internalized (by receptor mediated endocytosis?) and accumulated in the reservosomes. As GSH may serve as a storage and transport form of cysteine moieties, it was suggested that the Tc52GSH complexes might act as a cysteine delivery system. Accordingly, TC52 is developmentally regulated, being fully expressed only by the epimastigotes.

The presence of an early endosomal compartment in T. cruzi epimastigotes is still controversial. It is well known that incubation of the para- 
sites at $28^{\circ} \mathrm{C}$ with gold labeled proteins results in labeling inside cytoplasmic vesicles and tubules, as well as in the reservosomes (Soares \& De Souza 1991, Soares et al. 1992). Figueiredo and Soares (1996) showed that incubation of the parasites at $12^{\circ} \mathrm{C}$ (a condition that hinders the fusion of endocytic vesicles with early endosomes in mammalian cells) blocked the pinching of endocytic vesicles at the cytostome, inhibiting the uptake of nutrients by the cells. Labeling could be found in the cytostome, but not inside the flagellar pocket or intracytoplasmic vesicles. When the temperature was raised to $28^{\circ} \mathrm{C}$, labeling could be then again found in the reservosomes. From these experiments, it was concluded that early endosomes are lacking; cargo vesicles coming from the cell surface (cytostome and flagellar pocket membranes) should shuttle their content directly to the reservosomes. On the other hand, three-dimensional reconstruction of cytoplasmic tubules and vesicles located close to the flagellar pocket showed that they are interconnected, forming a branched network at the anterior end of the cell, morphologically similar to the typical mammalian early endosomes (Porto-Carreiro et al. 1998).

Bogitsh et al. (1996) presented some data demonstrating that, although containing cysteine proteinase, reservosomes are unlikely to be lysosomes (albeit lysosomes have not yet been clearly morphologically and biochemically defined in trypanosomatids). The authors showed that incubation of epimastigotes with ammonium chloride (a weak base that accumulates in acidic compartments) resulted in swelling of reservosomes and electron-lucent vacuoles (considered as lysosomes). However, the exposure period required for swelling of reservosomes was significantly greater than that required for the same effect in lysosomes, probably due to the different $\mathrm{pH}$ inside these compartments. Furthermore, methyl esters of aminoacids (which accumulate in eukaryotic lysosomes) had little effect upon reservosomes, precluding their being lysosomes and suggesting that the proteolytic enzymes, such as cysteine proteinases, can be in an inactive state during a life period of the parasites.

\section{THE ROLE OF RESERVOSOMES IN METACYCLO- GENESIS}

A stereological study showed that reservosomes occupy about $6 \%$ of the total cell volume of epimastigotes, but gradually vanish during the differentiation process to the trypomastigote form (Soares et al. 1989, Figueiredo et al. 1994). It has been suggested that the nutrients accumulated in the reservosomes could be used as a main energy source for this activity. A fascinating hypothesis is that a nutritional stress triggers the acidification of the luminal content and the activation of the enzymes contained inside the reservosomes, which then evolve to a lysosomal state. Degradation of stored proteins would then lead to the disappearance of the reservosomes, with the release of amino acids to the cell cytoplasm. Accordingly, biochemical data demonstrate that consumption of amino acids is favored in epimastigotes under starvation conditions (Urbina 1994).

Reservosomes contain cruzipain (also known as cruzain and GP57/51), the major cysteine proteinase of T. cruzi. Expression of cruzipain is developmentally regulated, the enzyme levels being about 10-fold higher in epimastigotes (Cazzulo et al. 1997). High expression of reservosomes and cysteine proteinases in epimastigotes, but not in trypomastigotes, suggests the participation of reservosomes in T. cruzi metacyclogenesis. Some data support this hypothesis: Franke de Cazzulo et al. (1994) demonstrated that proteinase inhibitors reduced growth and differentiation of T. cruzi. Ultrastructural data showed that treatment of the parasites with cysteine proteinase inhibitors arrested the transport of the enzymes to the reservosomes at the Golgi complex cisternae level, leading to cell death (Engel et al. 1998). Preliminary observations reported by Figueiredo et al. (1998) in epimastigotes maintained in TAU3AAG medium (a condition that induces metacyclogenesis) showed that a close relationship exists between uptake of nutrients, adhesion to the substrate and cell differentiation in T. cruzi.

\section{CONCLUSION}

Although the reservosome seems to play a pivotal role in the life cycle of $T$. cruzi, little is still known about this fascinating organelle. A still blurry image is slowly coming to sight relative to the endocytic process of $T$. cruzi cells, but unfortunately most data has been obtained from epimastigote forms maintained in culture media. A more precise characterization of reservosomes and cytoplasmic vesicles is at the moment difficult, since specific markers to the endocytic compartments are still lacking. Some hope comes from the recent obtention of a purified subcellular fraction containing reservosomes of $\mathrm{T}$. cruzi epimastigotes (Cunha-e-Silva et al. 1998). Involvement of this organelle in vital metabolic pathways of the parasites indicates that reservosomes are potential targets for the development of chemotherapeutic drugs.

\section{REFERENCES}

Benchimol M, De Souza W, Vanderheyden N, Zhong L, Lu H-G, Moreno SNJ 1998. Functional expression 
of a vacuolar-type $\mathrm{H}^{+}$-ATPase in the plasma membrane and intracellular vacuoles of Trypanosoma cruzi. Biochem J 332: 695-702.

Bogitsh BJ, Ribeiro-Rodrigues E, Carter CE 1997. The in vitro effects of extracellular adenosine triphosphate on the ultrastructure of Trypanosoma cruzi epimastigotes. Parasitol Res 83: 624-626.

Cazzulo JJ, Stoka V, Turk V 1997. Cruzipain, the major cysteine proteinase from the protozoan parasite Trypanosoma cruzi. Biol Chem 378: 1-10.

Cunha-e-Silva NL, Morgado-Diaz J, Porto-Carreiro I, De Souza W 1998. Obtention of a purified sub-cellular fraction containing reservosomes of Trypanosoma cruzi. Mem Inst Oswaldo Cruz, 93(Suppl. II): Abstract BI-128.

De Souza W 1984. Cell biology of Trypanosoma cruzi. Int Rev Cytol 86: 197-285.

Engel JC, Doyle PS, Palmer J, Hsieh I, Bainton DF, McKerrow JH 1998. Cysteine protease inhibitors alter Golgi complex ultrastructure and function in Trypanosoma cruzi. J Cell Sci 111: 597-606.

Figueiredo RCBQ, Soares MJ 1996. Low temperature inhibits the uptake of transferrin-gold complexes by epimastigote forms of Trypanosoma cruzi. Mem Inst Oswaldo Cruz 91(Suppl. I): 214.

Figueiredo RCQ, Rosa DS, Soares MJ 1998. Nutritional stress regulates adhesion to substrate and metacyclogenesis in Trypanosoma cruzi. Mem Inst Oswaldo Cruz, 93 (Suppl. II): 91.

Figueiredo RCBQ, Steindel M, Soares MJ 1994. The reservosomes of epimastigote forms of Trypanosoma cruzi: occurrence during in vitro cultivation. Parasitol Res 80: 517-522.

Franke de Cazzulo BM, Martínez J, North MJ, Coombs GH, Cazzulo JJ 1994. Effects of proteinase inhibitors on the growth and differentiation of Trypanosoma cruzi. FEMS Microbiol Lett 124: 81-86.

Okuda K, Esteva M, Segura E, Bijovsky AT 1997. The cytostome of Trypanosoma cruzi epimastigotes is a cytoskeletal structure associated to the flagellar complex. Mem Inst Oswaldo Cruz 92 (Suppl. I): 88.

Ouassi MA, Dubremetz JF, Schöneck R, FernandezGomez R, Gomez-Corvera R, Billaut-Mulot O, Taibi
A, Loyens M, Tartar A, Sergheraert C, Kusnierz JP 1995. Trypanosoma cruzi: a 52-kDa protein sharing homology with glutathione S-transferase is localized in parasite organelles morphologically resembling reservosomes. Exp Parasitol 81: 453-461.

Overath P, Stierhof Y-D, Wiese M 1997. Endocytosis and secretion in trypanosomatid parasites - tumultuous traffic in a pocket. Trends Cell Biol 7: 27-33.

Porto-Carreiro I, Attias M, Sant'Anna C, De Souza W, Cunha-e-Silva N 1998. Trypanosoma cruzi epimastigotes endocytic pathway: cargo enters the cytostome and passes through an early endosomal network before reservosome storage. Mem Inst Oswaldo Cruz 93 (Suppl. II): Abstract BI-134.

Radek R, Hausmann K 1994. Endocytosis, digestion, and defecation in flagellates. Acta Protozool 33: 127 147.

Scott DA, Docampo R, Dvorak JA, Shi S, Leapman RD 1997. In situ compositional analysis of acidocalcisomes in Trypanosoma cruzi. J Biol Chem 44: 28020-28029.

Soares MJ, De Souza W 1988. Cytoplasmic organelles of trypanosomatids: a cytochemical and stereological study. J Submicrosc Cytol Pathol 20: 349-361.

Soares MJ, De Souza W 1991. Endocytosis of gold-labeled proteins and LDL by Trypanosoma cruzi. Parasitol Res 77: 461-468.

Soares MJ, Souto-Padrón T, Bonaldo MC, Goldenberg S, De Souza W 1989. A stereological study of the differentiation process in Trypanosoma cruzi. Parasitol Res 75: 522-527.

Soares MJ, Souto-Padrón T, De Souza W 1992. Identification of a large pre-lysosomal compartment in the pathogenic protozoon Trypanosoma cruzi. J Cell Sci 102: $157-167$.

Urbina JA 1994. Intermediary metabolism of Trypanosoma cruzi. Parasitol Today 10: 107-110.

Vieira M, Carvalho T, Souto-Padrón T, Cunha-e-Silva N, De Souza W 1996. Tyrosine-phosphorylated proteins are present in Trypanosoma cruzi reservosomes. Mem Inst Oswaldo Cruz 91(Suppl. I): 213.

Webster P, Russel DG 1993. The flagellar pocket of trypanosomatids. Parasitol Today 9: 201-205. 